\title{
Histone demethylase JARID1C inactivation triggers genomic instability in sporadic renal cancer
}

\author{
Beatrice Rondinelli, ${ }^{1,2}$ Dalia Rosano, ${ }^{1}$ Elena Antonini, ${ }^{1}$ Michela Frenquelli, ${ }^{1}$ Laura Montanini, ${ }^{2}$ DaChuan Huang, ${ }^{3}$ Simona Segalla, \\ Kosuke Yoshihara, ${ }^{4}$ Samir B. Amin, ${ }^{5}$ Dejan Lazarevic, ${ }^{6}$ Bin Tean The, ${ }^{3}$ Roel G.W. Verhaak, ${ }^{4,5}$ P. Andrew Futreal, ${ }^{5}$ Luciano Di Croce, ${ }^{7,8}$ \\ Lynda Chin, ${ }^{5}$ Davide Cittaro, ${ }^{6}$ and Giovanni Tonon ${ }^{1}$ \\ ${ }^{1}$ Functional Genomics of Cancer Unit, Department of Experimental Oncology, Istituto di Ricovero e Cura a Carattere Scientifico (IRCCS), San Raffaele Scientific Institute, Milan, Italy. \\ ${ }^{2}$ Vita-Salute San Raffaele University, Milan, Italy. ${ }^{3}$ Laboratory of Cancer Epigenome, Division of Medical Sciences, National Cancer Centre Singapore, Singapore. ${ }^{4}$ Department of Bioinformatics and \\ Computational Biology and ${ }^{5}$ Department of Genomic Medicine, The University of Texas MD Anderson Cancer Center, Houston, Texas, USA. ${ }^{6}$ Centre for Translational Cenomics and Bioinformatics, \\ IRCCS, San Raffaele Scientific Institute, Milan, Italy. 'entre for Cenomic Regulation (CRG), Universitat Pompeu Fabra, Barcelona, Spain. \\ ${ }^{8}$ Institució Catalana de Recerca i Estudis Avançats (ICREA), Barcelona, Spain.
}

\begin{abstract}
Mutations in genes encoding chromatin-remodeling proteins are often identified in a variety of cancers. For example, the histone demethylase JARID1C is frequently inactivated in patients with clear cell renal cell carcinoma (ccRCC); however, it is largely unknown how JARID1C dysfunction promotes cancer. Here, we determined that JARID1C binds broadly to chromatin domains characterized by the trimethylation of lysine 9 (H3K9me3), which is a histone mark enriched in heterochromatin. Moreover, we found that JARID1C localizes on heterochromatin, is required for heterochromatin replication, and forms a complex with established players of heterochromatin assembly, including SUV39H1 and HP1 $\alpha$, as well as with proteins not previously associated with heterochromatin assembly, such as the cullin 4 (CUL4) complex adaptor protein DDB1. Transcription on heterochromatin is tightly suppressed to safeguard the genome, and in ccRCC cells, JARID1C inactivation led to the unrestrained expression of heterochromatic noncoding RNAs (ncRNAs) that in turn triggered genomic instability. Moreover, ccRCC patients harboring JARID1C mutations exhibited aberrant ncRNA expression and increased genomic rearrangements compared with ccRCC patients with tumors endowed with other genetic lesions. Together, these data suggest that inactivation of JARID1C in renal cancer leads to heterochromatin disruption, genomic rearrangement, and aggressive ccRCCs. Moreover, our results shed light on a mechanism that underlies genomic instability in sporadic cancers.
\end{abstract}

\section{Introduction}

The role of chromatin remodelers in regulating gene expression has been established (1). Prominent examples include the polycomb group $(\mathrm{PcG})$ and trithorax group (TrxG) proteins that compete to respectively constrain or enable the transcriptional machinery to access to genes and genomic elements (2). Indeed, beyond promoters, other genomic elements are enlisted in this layered regulation, including enhancers (3), or, within the gene bodies, introns and exons (4), ultimately affecting a myriad of locations scattered throughout the genome. As a result, these chromatin modifiers directly or indirectly coordinate deeply intertwined developmental and cell-signaling programs (5).

Chromatin remodelers exert additional, transcription-independent roles in DNA protection and maintenance, acting on large DNA domains and ultimately shaping the architecture and anatomy of the genome on a more global level (6). As an example, during physiological differentiation, large swaths of DNA become compacted into heterochromatin domains, thus preserving genomic stability and repressing the transcription of potentially harmful repetitive DNA sequences (7).

Authorship note: Dalia Rosano and Elena Antonini contributed equally to this work Conflict of interest: The authors have declared that no conflict of interest exists. Submitted: January 16, 2015; Accepted: October 8, 2015.

Reference information: J Clin Invest. 2015;125(12):4625-4637. doi:10.1172/JCI81040.
Heterochromatin exists in two forms, facultative and constitutive. Facultative heterochromatin is interspersed within chromosomes and mediates context-dependent, transient epigenetic silencing of genes. It has been implicated in developmental programming, cell fate determination, mating-type gene silencing, and $\mathrm{X}$ chromosome inactivation (8). Conversely, constitutive heterochromatin is present at sites marked by repetitive genetic elements, including telomeres and centromeres. As for its functions, the highly condensed heterochromatic structure at these genomic sites limits the access of transcription and recombination machineries, thus protecting the genome from unwanted, potentially harmful transposition events (9). In addition, at centromeres, the presence of this compact structure is crucial for kinetochore formation and, ultimately, chromosome segregation (10). Posttranslational histone modifications are fundamental for maintaining centromeric heterochromatin in a highly condensed structure. These modifications are remarkably well conserved across species $(11,12)$. Among these are di- or trimethylated lysine 9 of histone $\mathrm{H} 3$ (H3K9me2/3) (13), trimethylated lysine 20 of histone H4 (H4K2Ome3) (14), and monomethylated lysine 27 of histone H3 (H3K27me) $(15,16)$.

Constitutive heterochromatin remains condensed during interphase. However, at the onset of DNA replication and similarly to the rest of the genome, heterochromatin needs to undergo duplication. This event entails a transient disruption of the het- 
erochromatin structure to allow movement of the replication fork. While the detailed mechanism responsible for its temporary disbanding remains largely unknown, several remodeling complexes contribute to heterochromatin restoration after DNA replication (17-19). The mechanisms involved in this process are well conserved across evolution. In fission yeast, the stepwise mechanism for heterochromatin restoration involves methylation at lysine 9 of histone 3 by the histone methyltransferase Clr4 (20). H3K9me3 is then recognized by chromodomain-containing proteins Chp2 and Swi6 (the yeast homologs for the HP1 $\alpha$ heterochromatin protein $1 \alpha$ ), which oligomerize upon binding to nucleosomes (21). Clr4 itself can dock to methylated H3K9 through its own chromodomain and methylate neighboring nucleosomes, thus generating additional binding sites for Swi6/HP1 proteins. Similarly to fission yeast, mouse centromeric and pericentromeric repeats are trimethylated at $\mathrm{H} 3 \mathrm{~K} 9$, in this instance by SUV39H1/2 methyltransferases (22-24). Subsequently, trimethylated lysine 9 is a docking site for HP1 proteins (25). Spreading of heterochromatin proceeds as a self-sustaining loop, where HP1 isoforms themselves recruit repressive DNA methyltransferases (13).

While H3K9me3 has been consistently associated with heterochromatin domains, another histone posttranslational modification, $\mathrm{H} 3 \mathrm{~K} 4 \mathrm{me}$, is enriched in transcriptionally active euchromatin (26). It has been recently proposed that histone demethylases targeting $\mathrm{H} 3 \mathrm{~K} 4 \mathrm{me} 3$ are also required for proper heterochromatin assembly, in both yeast (Lid2) (27) and Drosophila [SU(VAR)3-3] (28), suggesting that removal of this histone mark from nascent heterochromatin is a required step toward its duplication. Remarkably, in higher eukaryotes, defective $\mathrm{H} 3 \mathrm{~K} 4$ trimethylation is associated with aberrant centromeric function, leading to chromosome misalignment and segregation defects (29). However, the mechanism underlying the removal of methyl marks from $\mathrm{H} 3 \mathrm{~K} 4$ on heterochromatin in vertebrates remains unknown.

Arguably, one of the main findings emerging from the recent flurry of next-generation sequencing efforts conducted for hundreds of cancer patients, including The Cancer Genoma Atlas (TCGA) initiative, is the remarkably high frequency of mutations affecting chromatin-related genes, indeed rivaling, in terms of occurrence, seasoned, established mutated tumor-suppressor genes and oncogenes such as RAS and TP53 (30). Prominent examples include SMARCB1 inactivation in rhabdoid tumors (31), ARID1A and ARID1B in endometrial tumors and neuroblastomas $(32,33)$, and the SWI/SNF subunit PBRM1 in clear cell renal cell carcinomas (ccRCCs) (34). Indeed, ccRCC represents a particularly cogent example of the anticipated oncogenic role of chromatin modifiers in cancer, since, alongside $V H L$, histone-modifying genes are by far the most frequently deleted genes in this disease. Specifically, SETD2/KMT3A, UTX/KDM6A, MLL2/KMT2B, and JARID1C/KDM5C (hereafter referred to as JARID1C) are, with variable frequencies, consistently mutated in renal cancer (35-37). In particular, JARID1C mutations have been reported in up to $9 \%$ of patients with ccRCC (35-37).

JARID1C resides on the $\mathrm{X}$ chromosome. It is expressed in multiple human tissues, has a paralog on the Y chromosome, and is highly conserved across evolution (38). JARID1C is one of the few genes on the $\mathrm{X}$ chromosome that escapes $\mathrm{X}$ inactivation (38). JARID1C missense, frame-shift, and nonsense mutations have been linked to X-linked mental retardation (XLMR) (39). Notably, most of the nucleotide substitutions identified so far in this disease cause partial or complete loss of function in the demethylase activity of the protein $(40,41)$. JARID1C plays a prominent role in neuronal development and function. Indeed, silencing of JARID1C expression in zebrafish derails neuronal development, including brain-patterning defects as well as impaired dendrite development and significant neuronal cell death in rat neurons (41).

JARID1C belongs to the JARID subfamily of JmjC-containing proteins, together with RBP2 (JARID1A), PLU-1 (JARID1B), and SMCY (JARID1D) (42). The JmjC domain of JARID1C represents its catalytic moiety that specifically demethylates di- and trimethylated lysine 4 on histone 3 in an Fe(II)- and $\alpha$-ketoglutaratedependent manner (41). The JmjN domain and the C5HC2 zinc finger are both important for assisting JARID1C catalytic activity (43). JARID1C also contains a BRIGHT domain and an AT-rich interaction domain (ARID) that can bind DNA (44). Finally, one of the two PHD domains binds trimethylated lysine 9 on histone 3 (H3K9me3) (41).

JARID1C exerts its neurodevelopmental role through transcriptional repression. In fact, it was isolated as part of a transcriptionally repressive complex containing HDAC1/2, EHMT2 (G9a), and REST (45). This complex binds the REST-responsive elements on neuronal-specific promoters such as brain-derived neurotrophic factor (BDNF), SCG10, and SCN2A, inhibiting their transcription through JARID1C demethylase activity.

Recent reports have demonstrated a prominent role of JARID1C in modulating H3K4me3 levels throughout the genome. In mouse embryonic stem cells and in neuronal progenitor cells, JARID1C is recruited on regulatory regions such as enhancers and promoters. Intriguingly, JARID1C restrains transcription, reducing $\mathrm{H} 3 \mathrm{~K} 4 \mathrm{me} 3$ at promoters, while it stimulates their activity at enhancers (46). Along similar lines, in renal cancer cell lines, JARID1C also comprehensively regulates H3K4me3 levels (47). Additionally, we have recently identified a nontranscriptional role for JARID1C in DNA replication (48).

Notwithstanding the frequent inactivation of JARID1C in ccRCCs, little is known about the underlying tumorigenic mechanisms. Here, we sought to provide insights on the role of JARID1C in ccRCCs and to identify the consequences of its inactivation in human cancers.

In this study, we demonstrate that JARID1C broadly binds to H3K9me3 heterochromatic chromatin domains and is required for heterochromatin replication in a complex with SUV39H1 and HP1 $\alpha$, as well as with the cullin 4 (CUL4) complex adaptor protein DDB1. JARID1C loss unleashes the expression of heterochromatic noncoding RNAs (ncRNAs), thus triggering genomic instability. Notably, ccRCC tumors presenting with JARID1C mutations are more genetically rearranged than ccRCC tumors with mutations in other driver genes.

\section{Results}

JARID1C binds to broad chromatin domains enriched for the H3K9me3 histone mark and to heterochromatin. In order to explore JARID1C function in renal cancer, we conducted a ChIP-sequencing (ChIP-seq) analysis, assaying the binding of JARID1C to chromatin. To this end, we used two commercially available Abs 
A
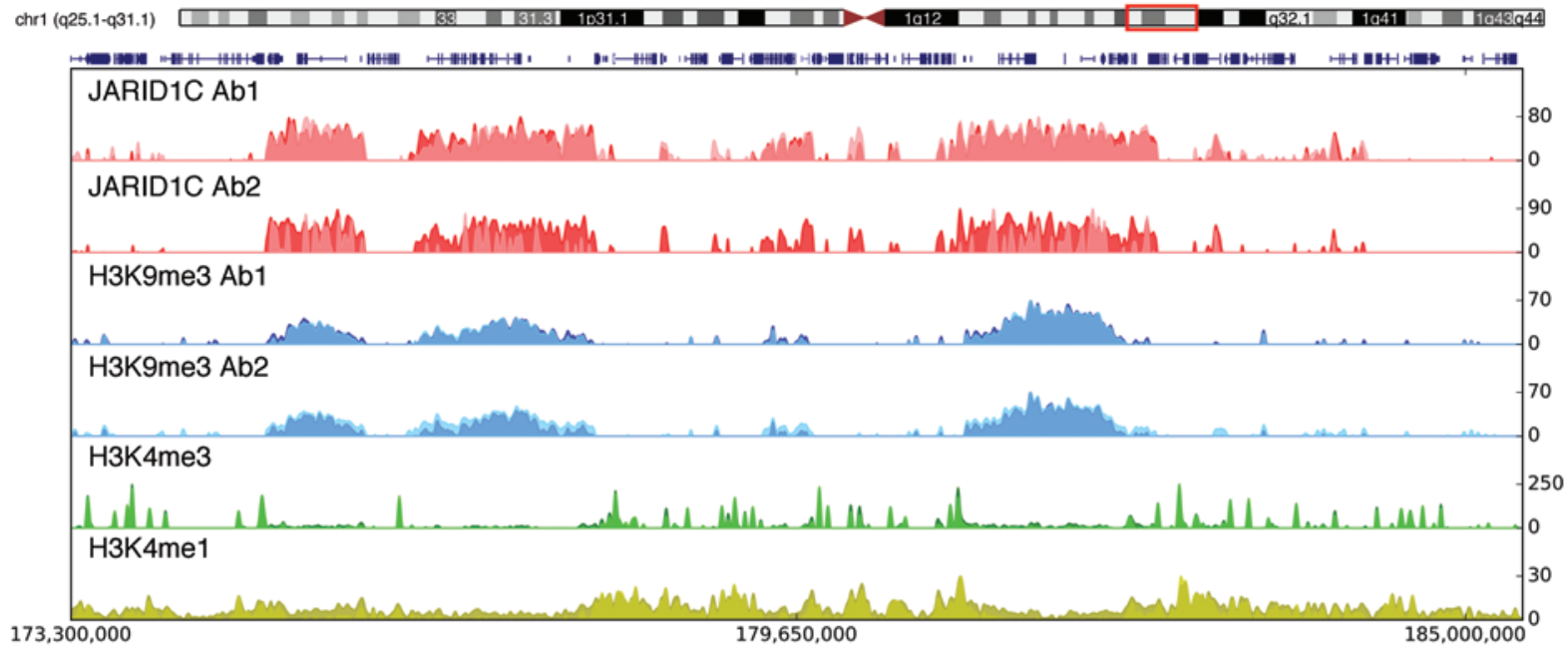

B
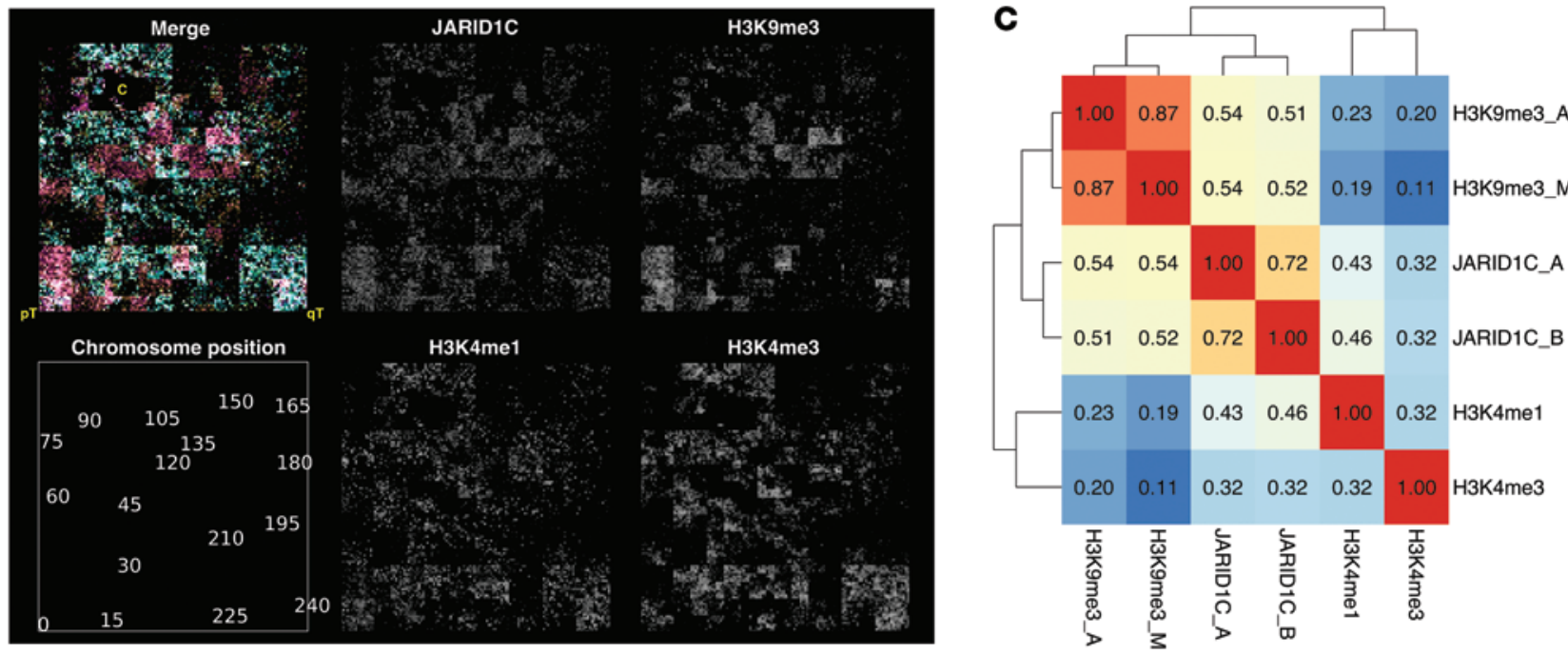

D

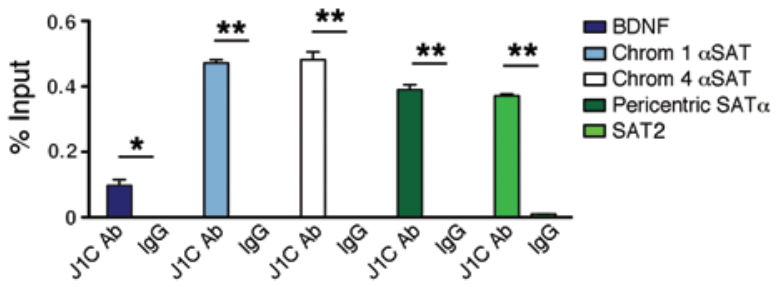

Figure 1. Co-occurrence of JARID1C and heterochromatin. (A) Snapshot of representative genomic region showing the co-occupancy of JARID1C and H3K9me3 ChIP-seq profiles. Two different Abs were used for the JARID1C (Ab1, Abcam; Ab2, Bethyl Laboratories) and H3K9me3 (Ab1, EMD Millipore; Ab2, Abcam) IP experiments. Each track represents an overlay of 2 independent replicates. chr1, chromosome 1. (B) Hilbert curve representation of multiple ChIP-seq profiles on chromosome 2. Multiple markers were assigned to the color channel before merging (JARID1C, yellow; H3K9me3, magenta; H3K4me1, cyan; H3K4me3, gray). Merged image is annotated with both telomere positions (pT, short arm; qT, long arm) and the centromere (c); a map of chromosome positions is also presented in the bottom left panel. (C) Heatmap showing the correlation between the multiple ChIP-seq signals discussed in the main text. Correlation values were used to generate dendrograms. (D) ChIP with anti-JARID1C Ab specific to the human protein in Caki-1 renal cancer cells. ChIP was analyzed by qPCR with primers for BDNF, a known promoter of binding sites for JARID1C (45), chrom 1 and chrom $4 \alpha$ SAT (centric satellites of chromosomes 1 and 4), and SAT2 and pericentric SAT $\alpha$, pericentric satellites of human chromosomes. Results are expressed as the percentage of input. Error bars represent the SEM of 3 independent experiments. ${ }^{*} P<0.05,{ }^{* *} P<0.01$, Student's $t$ test.

raised against JARID1C. We also assayed for established histone modifications, including $\mathrm{H} 3 \mathrm{~K} 4 \mathrm{me} 1, \mathrm{H} 3 \mathrm{~K} 4 \mathrm{me} 3$, and H3K9me3. In line with previous reports $(46,49)$, JARID1C ChIP-seq profiles showed narrow peaks at regions corresponding to promoters and enhancers. Surprisingly, JARID1C also bound additional regions of the genome, showing a broader binding pattern (Figure 1, A and B, and Supplemental Figure 1A; supplemental material available online with this article; doi:10.1172/JCI81040DS1). While sharp JARID1C signals colocalized with $\mathrm{H} 3 \mathrm{~K} 4 \mathrm{me} 3$ and $\mathrm{H} 3 \mathrm{~K} 4 \mathrm{me} 1$ peaks $(P<0.01)$, JARID1C broader features were significantly associated 
either with H3K4me1 or, surprisingly, with H3K9me3 domains ( $P$ $<0.01$; Figure 1, A and B). Indeed, the JARID1C ChIP-seq profile correlated most strongly with $\mathrm{H} 3 \mathrm{~K} 9 \mathrm{me} 3$ tracks (range, $r=0.51-$ 0.54) (Figure 1C and Supplemental Figure 1B). This association was confirmed in ChIP-seq experiments using a second $\mathrm{Ab}$ recognizing H3K9me3 (Figure 1A and Supplemental Figure 1B). To further validate these results, we analyzed a data set of the human leukemia cell line K562 that included ChIP-seq profiles of a large number of transcription factors, chromatin modifiers, and histone marks (49). We also found in this data set that the JARID1C ChIPseq profile was strongly associated with H3K9me3 (Supplemental Figure 1C). Altogether, these data suggest that JARID1C colocalizes on the genome with H3K9me3.

Since H3K9me3 is the hallmark histone mark of heterochromatin, we next explored whether JARID1C localizes on established heterochromatin sequences. ChIP experiments demonstrated a significant enrichment of JARID1C at pericentric (pericentric SAT $\alpha$ and SAT 2 ) and centromeric (chrom 1 and chrom $4 \alpha$ SAT) sequences as compared with that observed in the isotypic control in renal cells (Figure 1D). In all, these results suggest that JARID1C colocalizes with $\mathrm{H} 3 \mathrm{~K} 9 \mathrm{me} 3$ and binds to heterochromatin domains.

JARID1C contributes to heterochromatin maintenance and directly binds to satellite repeats during heterochromatin duplication in the $S$ phase. We next sought to determine whether JARID1C might have a role in heterochromatin assembly. To this end, we exploited $\mathrm{NIH}-3 \mathrm{~T} 3$ cells, the model system of choice for assessing heterochromatin status (50). Indeed, the downregulation of JARID1C with two different shRNAs significantly decreased the overall number of DAPI-positive heterochromatic centers (Figure 2, A and $\mathrm{B}$, left; on average, from 20.49 to 16.67 and 15.65 foci/cell respectively; $P<0.0001)$. Furthermore, the DAPI foci appeared significantly larger (Figure 2, A and B, right). Altogether, these data suggest that JARID1C participates in heterochromatin maintenance.

Heterochromatin assembly occurs during the middle and late stages of the S phase $(51,52)$. In NIH-3T3 cells, it has been reported that S-phase progression could be evaluated by assaying the distribution of nuclear DNA replication sites by BrdU-specific immunofluorescence $(53,54)$. Specifically, in the early $S$ phase, cycling cells present small and discrete BrdU-positive granules interspersed throughout the nucleus, while middle S-phase cells show fewer foci overlapping with heterochromatin domains. Ultimately, in late S phase, large, often irregular BrdU-positive dots are detected throughout the nucleus. Remarkably, we found that upon JARID1C depletion, there was a significant increase of more than 2-fold in cells showing a middle S-phase pattern compared with control cells (Figure 2, C and D; $P<0.001$ ). Confocal microscopic experiments with a flagged form of JARID1C (Supplemental Figure 2A) revealed extensive colocalization of JARID1C with DAPI-intense chromocenters in S-phase cells (Figure 2E and Supplemental Figure 2, B and C). Remarkably, JARID1C localization with chromocenters coincided with BrdU staining (Figure $2 \mathrm{E}$ and Supplemental Figure 2, B and C). Together, these results suggest a prominent role for JARID1C in heterochromatin assembly.

Loss of heterochromatic histone marks at pericentric and centromeric repeats of JARID1C-silenced cells. Transcriptionally poised euchromatin is enriched for H3K4me3 (26) and depleted of H3K9me3 (55). Heterochromatin displays the opposite pattern
$(20,56)$ in both yeast and mouse cells $(56-58)$. JARID1C is a histone demethylase that selectively demethylates H3K4me3 (41), a histone mark that is usually absent on heterochromatic satellites (57). We therefore reasoned that upon JARID1C inactivation, a disruptive enrichment of $\mathrm{H} 3 \mathrm{~K} 4 \mathrm{me} 3$ on heterochromatin might ensue. We first confirmed on whole-cell lysates that JARID1C downregulation was able to increase $\mathrm{H} 3 \mathrm{~K} 4 \mathrm{me} 3$ levels (Figure 3A). Moreover, H3K4me3 levels increased specifically at promoters targeted directly by JARID1C (ref. 45 and Supplemental Figure $3 \mathrm{~A})$. As for heterochromatin, ChIP experiments indeed revealed a robust increase in $\mathrm{H} 3 \mathrm{~K} 4 \mathrm{me} 3$ on centromeric (minor) and pericentric (major) regions after downregulation of JARID1C (Figure 3B). Notably, we did not detect this enrichment in other repeat sequences residing outside heterochromatin, such as SINEB1; nor did we observe changes on heterochromatin in other histone marks unrelated to JARID1C activity, such as H3K4me1 (Supplemental Figure 3B). Again, a similar aberrant methylation pattern affecting $\mathrm{H} 3 \mathrm{~K} 4$ was observed at pericentric and centromeric repeats in ccRCC cells upon downregulation of JARID1C (Supplemental Figure 3C). Moreover, exploiting another data set (46), JARID1C knockdown caused a significant increase in $\mathrm{H} 3 \mathrm{~K} 4 \mathrm{me} 3$ binding specifically on heterochromatic regions, confirming that the lack of JARID1C impairs H3K4me3 demethylation on heterochromatin (Supplemental Figure 3D).

Trimethylation of lysine 9 on histone 3 (H3K9) represents the hallmark histone modification on heterochromatin (20). H3K9me3 and H3K4me3 are mutually exclusive, both in vitro (59) and in vivo $(26,56)$. Remarkably, Western blotting on wholecell lysates showed a marked reduction of H3K9me3 following JARID1C downregulation (Figure 3A). We then explored whether JARID1C knockdown impaired the deposition of H3K9me3 on heterochromatin. Indeed, we tested whether JARID1C inactivation reduced $\mathrm{K} 9$ methylation specifically on heterochromatic satellite repeats using ChIP and observed a significant reduction of H3K9me3 on both minor and major repeats after JARID1C downregulation (Figure 3B). In addition, immunofluorescence revealed that the number of cells positive for $\mathrm{H} 3 \mathrm{~K} 9 \mathrm{me} 3$ chromocentric foci was significantly reduced (from $70.5 \% \pm 4 \%$ to $51 \% \pm 3 \% ; P<0.01$ ) in silenced cells (Figure 3C).

Importantly, rescue experiments on silenced cells demonstrated that WT, but not demethylase-dead mutant JARID1C, restored low $\mathrm{H} 3 \mathrm{~K} 4$ methylation levels at centric and pericentric repeats (Figure 3D) upon reexpression at comparable levels (Supplemental Figure 3, E-H), suggesting that the demethylase function of JARID1C is required for heterochromatin assembly in mammalian cells. Altogether, these data suggest that JARID1C actively removes methyl groups from $\mathrm{H} 3 \mathrm{~K} 4 \mathrm{me} 3$ on heterochromatic satellites and that loss of its demethylase function induces aberrant decompaction of heterochromatin structure as shown by aberrantly low $\mathrm{H} 3 \mathrm{~K} 9$ methylation levels.

JARID1C, together with DDB1, belongs to a heterochromatinsilencing complex and acts upstream of HP1 $\alpha$ and SUV39H1. Heterochromatin assembly includes several steps centered on trimethylation of H3K9me by SUV39H1 and DNA binding of $\mathrm{HP} 1 \alpha$, which ultimately stabilizes heterochromatin and favors its spreading $(25,60)$. Given the role of JARID1C in heterochromatin assembly, we next ascertained whether JARID1C interacts with 
A CTRsh

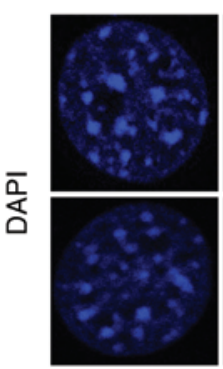

C $\square$ Early

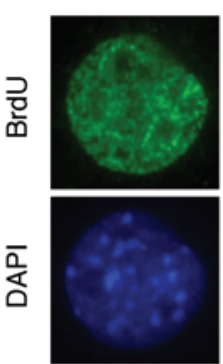

E

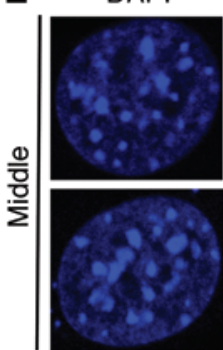

J1CshA/B
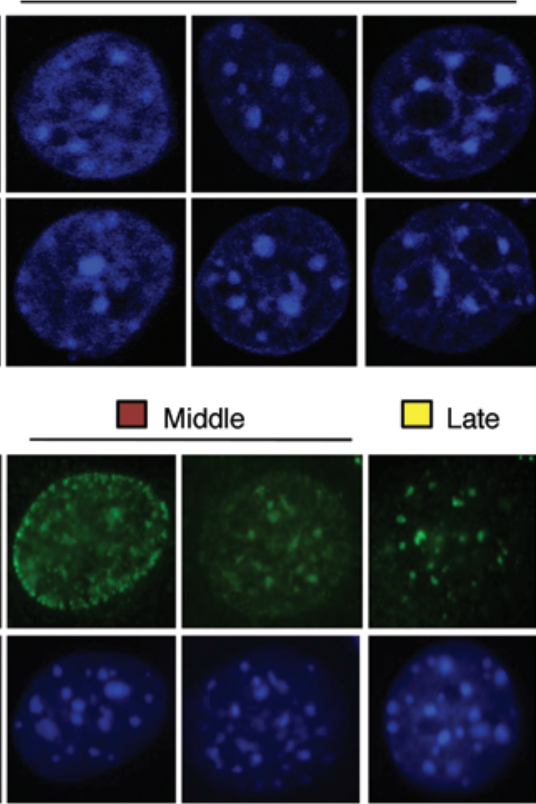

Middle

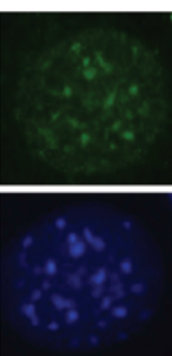

J1C

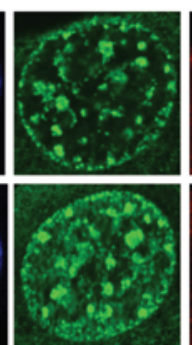

BrdU

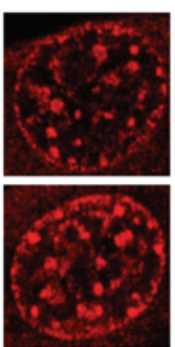

B

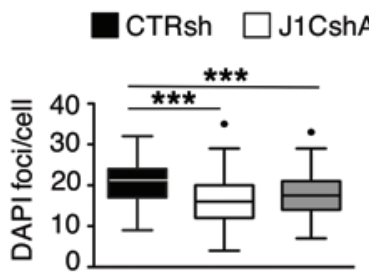

J1CshB

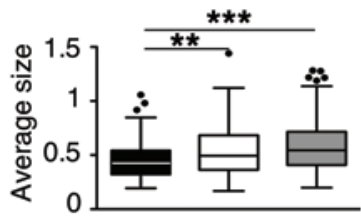

D
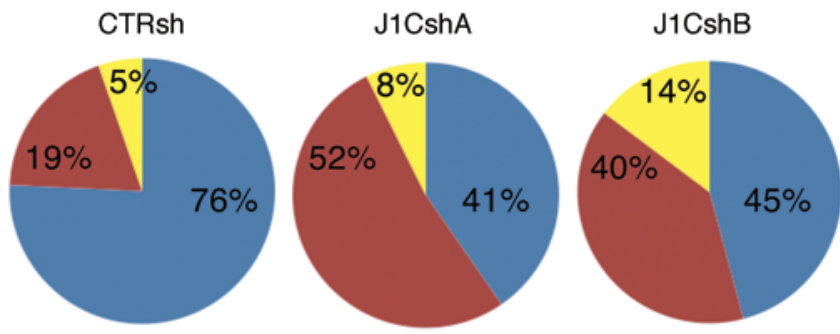

Figure 2. JARID1C contributes to heterochromatin maintenance and directly binds to satellite repeats during heterochromatin duplication. (A) JARID1C downregulation reduces the average number and increases the size of chromocenters per cell. Representative confocal DAPI images of interphase NIH-3T3 transfected with scrambled (CTRsh) or JARID1C-specific (J1CshA/B) shRNAs. (B) DAPI-stained nuclear foci per cell were counted and their average area assessed (square pixels $\times 10^{5}$ ). Data shown are from 1 of 3 experimental replicates. ${ }^{*} P<0.01$ and ${ }^{* * *} P<0.0001$, 1 -way ANOVA, with Tukey's multiple comparisons test. (C) Representative immunofluorescence images of NIH-3T3 cells stained for DAPI and BrdU. Typical early, middle, and late S-phase patterns are shown. (D) Pie charts showing quantitative analysis of the proportion of S-phase cells in early, middle, and late $S$ phase following transfection with control shRNA or shRNA against JARID1C. S-phase patterns were scored by BrdU patterns as shown in D. Data shown are from 1 of 2 experimental replicates ( $n=150 \mathrm{~S}$-phase nuclei per experiment). $P<0.001$, Pearson's $\chi^{2}$ test over the distribution. (E) Confocal immunofluorescence images of BrdU $\mathrm{NIH}-3 \mathrm{~T} 3$ cells transfected with JARID1C-FLAG. J1C represents the signal obtained with anti-FLAG Ab.

these proteins. Remarkably, co-IP experiments demonstrated a strong reciprocal binding between JARID1C and HP1 $\alpha$ (Figure 4A and Supplemental Figure 4A) as well as between JARID1C and SUV39H1 in renal cells (Figure 4B and Supplemental Figure 4B) and mouse cells (data not shown).

In Schizosaccharomyces pombe (S. pombe), H3K9me methylation on heterochromatin is promoted by the Dos1-Dos2-Rik1 complex $(61,62)$. Although Dos1 and Dos2 show weak similarity to WD-repeat proteins and zinc finger proteins, respectively, homologs in other organisms have not been described. Instead, it has been proposed that Rik1 is structurally related to the CUL4 complex adaptor protein DDB1 (63) through its WD-propeller-repeat domains $(64,65)$, despite limited primary sequence homology. Intriguingly, Rik1 is required during the $S$ phase to establish heterochromatin $(62,66)$. We thus asked whether DDB1 is part of the JARID1C-HP1 $\alpha$-SUV39H1 complex. Indeed, DDB1 immunopre- cipitated with both JARID1C (Figure 4C and Supplemental Figure 4C) and HP1 $\alpha$ (Supplemental Figure 4A), suggesting a potential role for human DDB1 in heterochromatin assembly, reminiscent of the role of yeast Rik1 (62). Altogether, these results indicate that JARID1C is part of a heterochromatin-remodeling complex in mammalian cells that includes HP1 $\alpha$ and SUV39H1 as well as the adaptor protein DDB1.

Given these protein-protein interactions and the reduced heterochromatic K9 methylation levels evident in JARID1C-downregulated cells, we reasoned that $\mathrm{K} 4$ demethylation induced by JARID1C might act upstream and be required for the engagement of both SUV39H1 and HP1 $\alpha$ to heterochromatin. Hence, we analyzed the binding to chromatin of both SUV39H1 and HP1 $\alpha$ upon JARID1C silencing. Notably, despite similar expression levels in control and downregulated cells (Figure 4D and Supplemental Figure 4D), SUV39H1 and HP1 $\alpha$ localization to heterochromatic 

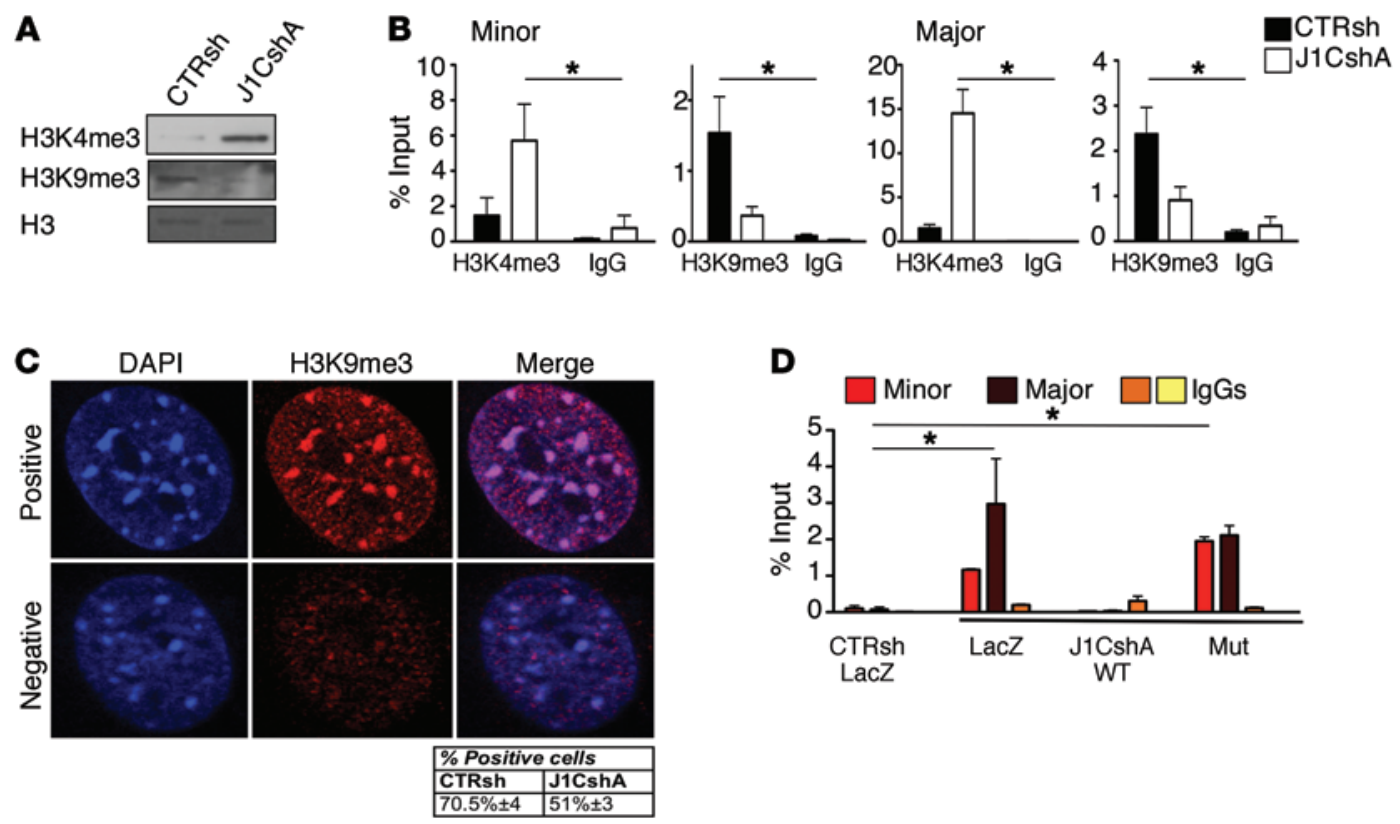

Figure 3. Loss of heterochromatic histone marks at pericentric and centromeric repeats of JARID1C-silenced cells. (A) Western blot analysis of total cell Iysates of CTRsh- and J1CshA-transfected NIH-3T3 cells. (B) Chromatin of control and silenced NIH-3T3 cells was immunoprecipitated with anti-H3K4me3and anti-H3K9me3-specific Abs and analyzed for enrichment by qPCR with specific minor and major primers, as listed in the Supplemental Methods. Isotypic IgGs were used as controls. Results are expressed as the percentage of input. Error bars represent the SEM of 3 independent experiments. ${ }^{*} P<0.05$, Student's $t$ test. (C) Confocal immunofluorescence images of NIH-3T3 cells stained with anti-H3K9me3-specific Ab and DAPI. Representative cells with heterochromatic $\mathrm{H} 3 \mathrm{~K} 9 \mathrm{me} 3$ dots (positive) and without dots (negative) are shown. The table scores the mean \pm SEM of 2 independent experiments (100 cells per condition analyzed; $P<0.01$, Fisher's exact test). (D) Rescue experiments were performed on NIH-3T3 cells, as reported in the Supplemental Methods. After 72 hours, chromatin was immunoprecipitated with an anti-H3K4me3-specific Ab. Enrichment was analyzed by qPCR with minor and major primers. Results are expressed as the percentage of input. Error bars represent the SEM of 3 independent replicates. Orange and yellow bars represent IgGs detected with minor and major qPCRs, respectively. ${ }^{*} P<0.05$, Student's $t$ test. Mut, mutant.

foci was significantly reduced in silenced cells (Figure 4, D and E, and Supplemental Figure 4E). We also ascertained whether HP1 $\alpha$ binding requires the demethylase activity of JARID1C. After JARID1C knockdown, we reintroduced mock, WT, or mutant JARID1C and performed ChIP to assess the binding of HP1 $\alpha$ to heterochromatin. Reintroduction of the JARID1C WT, but not the mutant, restored HP1 $\alpha$ heterochromatin binding (Figure 4F). These results suggest that JARID1C is required for SUV39H1 to localize to heterochromatin and methylate H3K9 and for HP1 $\alpha$ to bind and finalize heterochromatin assembly.

JARID1C loss deregulates heterochromatic noncoding RNAs, triggering genomic instability. Defects in heterochromatin assembly are linked to the persistent expression of ncRNAs (52). Therefore, we assayed for the presence of aberrantly transcribed heterochromatic pericentric (major) and centromeric (minor) ncRNAs in JARID1C-downregulated NIH-3T3 cells with both shRNAs. Notably, both minor and major satellite transcripts were significantly upregulated after JARID1C downregulation (Figure 5, A and B, and Supplemental Figure 5A). However, expression levels of other nonheterochromatic ncRNAs, such as LINEL1, SINEB1, and SINEB2, were not affected. A similar inordinate expression of heterochromatic ncRNAs was also detected in ccRCC cells upon JARID1C downregulation. In fact, the assessment of human SAT $\alpha$ and chrom 1 aSAT RNAs, corresponding to the mouse pericentric and centromeric transcripts, revealed a prominent increase in both species after JARID1C downregulation (Supplemental Figure 5, B and C, and data not shown).
To assess the role of JARID1C demethylase activity in heterochromatic ncRNA silencing, we designed a rescue experiment in which WT or demethylase-inactive (Mut; Figure 5C) JARID1C was introduced into cells knocked down for JARID1C. Remarkably, the WT, but not the demethylase-inactive isoform, was able to reduce expression levels of the minor and major heterochromatic satellites (Figure 5C), suggesting that silencing of pericentric and centromeric heterochromatic ncRNAs by JARID1C is mediated by its demethylase activity on H3K4me3.

Dysregulated expression of these RNA species embedded within heterochromatic regions leads to genomic instability (67). More generally, conditions associated with impaired heterochromatin formation have been causally linked to increased instability (24). To determine whether JARID1C downregulation was associated with this phenotype, we knocked down JARID1C expression in primary mouse embryonic fibroblasts (MEFs). Similarly to the other cell types, S-phase progression was derailed and BrdU incorporation reduced (Supplemental Figure 5, D and E). DAPI and $\alpha$-tubulin immunofluorescence staining of early-passage (P4/P6) silenced and control MEFs revealed significantly higher rates of bi- and trinucleation, misaggregation, and micronucleation in knocked-down cells compared with rates observed in control cells (Figure 5D). To conclusively demonstrate that the genomic instability ensuing as a result of JARID1C inactivation is mediated by the dysregulated expression of satellite repeats, we exploited locked nucleic acid (LNA) gapmers targeting minor and 
A

$$
\text { IP: J1C }
$$

IB: J1C

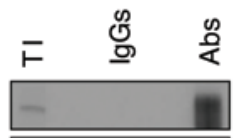

IB: HP1a

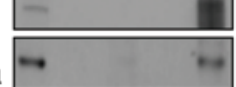

B

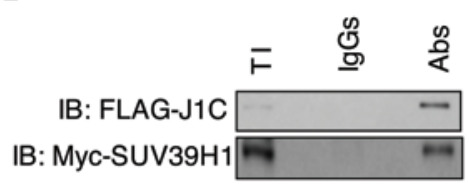

C

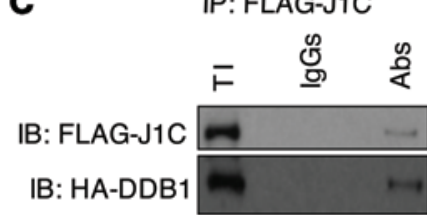

D
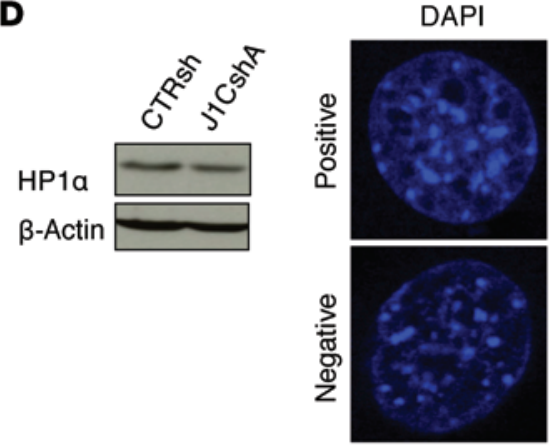
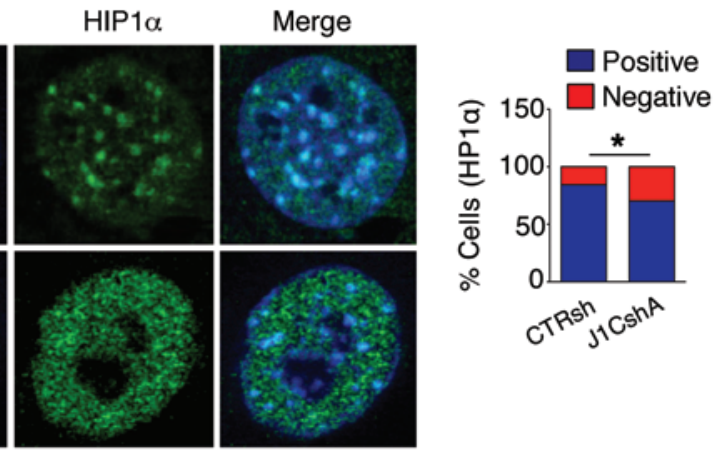

E

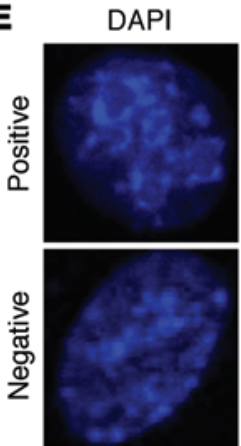

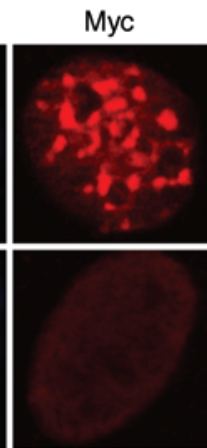
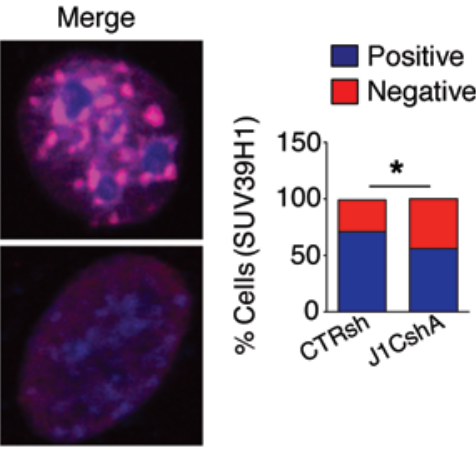

$\mathbf{F}$

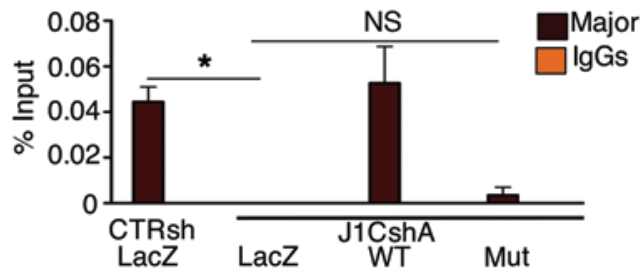

Figure 4. JARID1C, together with DDB1, belongs to a heterochromatin-remodeling complex and acts upstream of HP1 $\alpha$ and SUV39H1. Co-IP of JARID1C (J1C) with (A) HP1 $\alpha$, (B) SUV39H1, and (C) DDB1 in Caki-1 renal cancer cells. (A) IP for JARID1C and Western blotting for JARID1C and HP1 $\alpha$, (B) for JARID1C and a Myc Ab against SUV39H1, and C) for JARID1C and an HA Ab against DDB1, respectively. (D) Left: Total cell lysates of control (CTRsh) and downregulated (J1CshA) $\mathrm{NIH}-3 \mathrm{~T} 3$ cells were analyzed by Western blotting for expression of JARID1C, HP1 $\alpha$, and $\beta$-actin. Middle: Representative confocal immunofluorescence images of NIH-3T3 cells stained with anti-HP1 $\alpha$-specific Ab and DAPI. Representative cells with (positive) or without (negative) heterochromatic HP1 $\alpha$ dots are shown. Right: Graphs represent quantification (percentage of cells) of positive and negative CTRsh- and J1CshA-transfected cells from 3 independent experiments. Approximately 150 cells per condition were counted in each experiment. ${ }^{*} P<0.05$, Fisher's exact test. (E) Confocal immunofluorescence images of control and silenced $\mathrm{NIH}-3 \mathrm{~T} 3$ cells transfected with Myc-tagged SUV39H1 and stained with anti-Myc-specific Ab and DAPI. Representative cells with heterochromatic Myc dots (positive) and without dots (negative) are shown. Graph represents quantification of CTRsh and J1CshA cells with heterochromatic Myc-SUV39H1 dots from 2 independent experiments. Approximately 100 cells per condition were counted in each experiment. ${ }^{*} P<0.05$, Fisher's exact test. (F) Chromatin of NIH-3T3 rescue experiment was immunoprecipitated with an anti-HP1 $\alpha$-specific Ab. Enrichment was analyzed by qPCR at major repeats using specific primers. Results are expressed as the percentage of input. Orange square represents ChIP performed with control IgGs. Error bars represent the SEM of 3 independent replicates. ${ }^{*} P<0.05$, Student's $t$ test. IB, immunoblot; TI, total input.

major transcripts (68). Indeed, quenching these satellite ncRNAs reduced the genomic instability triggered by JARID1C knockdown in MEFs (Figure 5, E and F, and Supplemental Figure 5F). In all, these results suggest that JARID1C depletion triggers genomic instability through the aberrant transcription of satellite repeats.

ccRCC patients who present with JARID1C mutations show features of heterochromatin disruption, genomic rearrangements, and poor prognosis. We next sought to determine whether the cancer genomes of patients presenting with inactivating mutations in JARID1C have features in line with heterochromatin disruption, aberrant ncRNA expression, and enhanced instability. Notably, most JARID1C mutations are truncating, frameshift, and nonsense mutations (Supplemental Figure 6). The remaining missense mutations reside in critical domains, modifying highly conserved residues. Samples mutated for JARIDIC showed a significant loss of DNA methylation throughout the genome (Figure 6, A and B) when compared with tumors not mutated for this gene or with normal renal tissue, further underscoring the role of JARID1C in heterochromatin compaction. Notably, the regions hypomethylated in JARID1C-mutated samples were associated with enhanced H3K4me1 (the final product of JARID1C activity) in the corresponding regions in normal renal tissue (Supplemental Figure 7A). We next explored a data set from TCGA, seeking ncRNAs dysregulated in ccRCC samples mutated for JARID1C. We identified 194 lincRNAs (FDR <0.25) specifically dysregulated in this patient group (Supplemental Table 1). Notably, these lincRNAs were all 
A
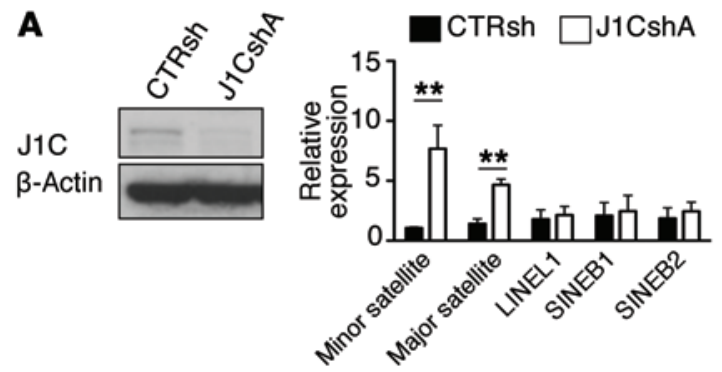

B

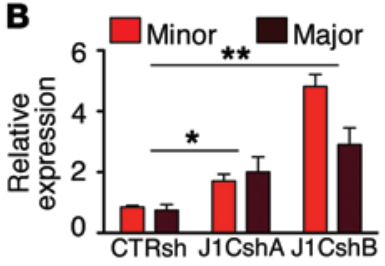

C

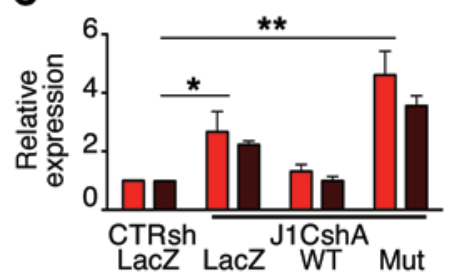

D
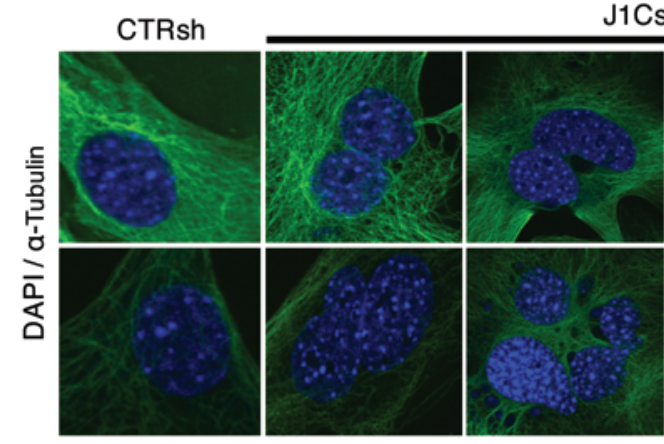

J1CshA/B

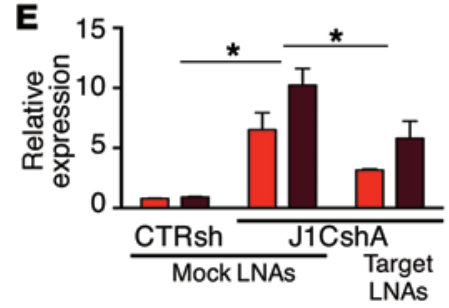

F 100
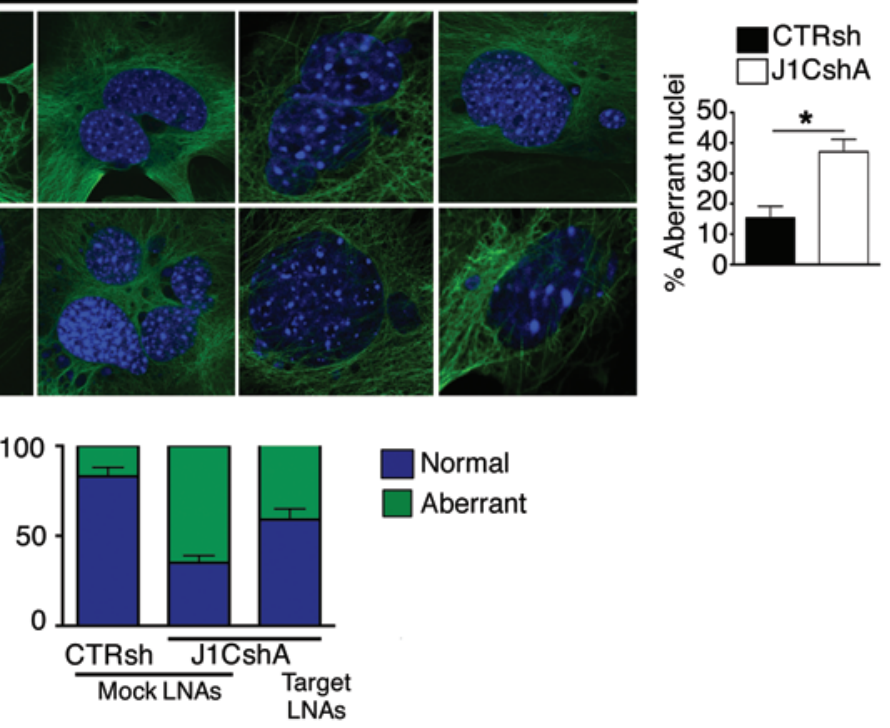

Figure 5. JARID1C loss deregulates expression of heterochromatic ncRNAs, triggering genomic instability. (A) Western blot analysis and qPCR expression of the indicated ncRNAs in NIH-3T3 cells knocked down with CTRsh or J1CshA. Results are expressed relative to GAPDH as the mean \pm SEM of 3 independent experiments. ${ }^{*} P<0.01$, Student's $t$ test. (B) Total RNA from CTRsh, J1CshA, or J1CshB NIH-3T3 cells was collected and ncRNAs expression analyzed by $q P C R$. Results are expressed relative to $G A P D H$ as the mean \pm SEM of 1 of 3 experimental replicates. (C) Rescue experiments were performed as described in the Supplemental Methods. Results are expressed relative to GAPDH. Bars depict 1 representative experiment of 5 independently performed experiments. ${ }^{*} P<0.05$ and ${ }^{*} P<0.01$, Student's $t$ test. (D) Left: Representative immunofluorescence images of early-passage (P2) MEFs transduced with CTRsh or J1CshA-specific lentiviruses and analyzed for DAPI and $\alpha$-tubulin (P5/P6). Right: Bars represent aberrant nuclei (see Supplemental Methods) in control and J1C-silenced MEFs, as quantified in 3 independent experiments (approximately 150 cells per condition for each experiment). Data are expressed as the mean \pm SEM. ${ }^{*} P<0.05$, Fisher exact test; $n=3$ experimental replicates. (E) Expression of minor and major satellite repeats in MEFs knocked down with CTRsh or J1CshA and subsequently transfected with a control sequence (Mock LNAs) or a mixture of LNAs targeting both minor and major transcripts (Target LNAs). Bars depict the mean \pm SEM of 3 independent experiments. ${ }^{*} P<0.05$, Student's $t$ test. (F) Stacked bar chart shows percentages of normal and aberrant nuclei in CTRsh- and J1CshA-transduced MEFs (see Supplemental Methods). Bars represent the mean \pm SEM of 3 independent experiments ( $n=150$ nuclei per condition per experiment). $P<0.001, \chi^{2}$ test over the distribution of the mean.

upregulated in JARID1C-mutated samples, further underscoring the repressive role of JARID1C in chromatin (Supplemental Table 1). Moreover, a direct correlation existed between lincRNA expression and the extent of $\mathrm{H} 3 \mathrm{~K} 9 \mathrm{me} 3 \mathrm{ChIP}$ peaks as detected in normal renal tissue, while an opposite pattern was evident for H3K 4me1 (Supplemental Figure 7, B and C). We also assayed for the presence of dysregulated heterochromatic ncRNAs directly on human ccRCC samples (see Supplemental Table 2 for sample information and mutation description) (37). Indeed, human SAT2 pericentric satellite RNA was significantly upregulated in tumor samples when compared with that in the corresponding normal tissues (Figure 6C). Intriguingly, Ting et al. have recently demonstrated a pervasive dysregulation of these satellite ncRNAs in several cancer types, including renal carcinomas (69). We further assessed whether the cancer genomes of patients presenting with inactivating mutations in JARID1C have features in line with enhanced instability. We thus analyzed 436 TCGA array comparative genomic hybridization (aCGH) profiles deposited in the cBioPortal for Cancer Genomics (70). Remarkably, the samples with JARID1C mutations showed a significantly higher number of copy number aberrations (CNAs) when compared with samples from the remaining ccRCC patients (Figure 6D). Specifically, most patients with JARID1C mutations presented with more than $10 \%$ of their genome rearranged, unlike that of patients in other subgroups, including those with SETD2 mutations, who, for the most part, presented with CNAs affecting less than $10 \%$ of their cancer genome (Figure 6D and data not shown). Importantly, patients presenting with JARIDIC-inactivating mutations have a significantly poorer prognosis when compared with patients without mutations (Supplemental Figure 7D). Together, these data 
A

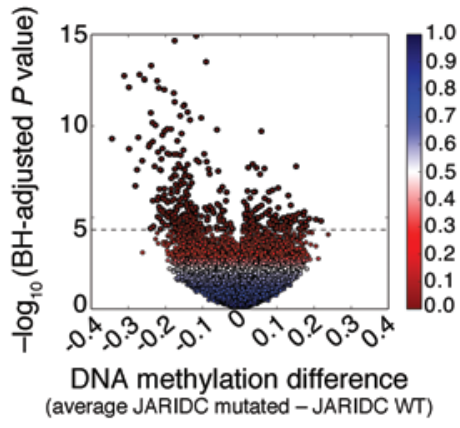

C

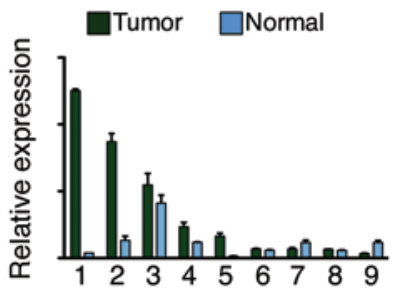

B

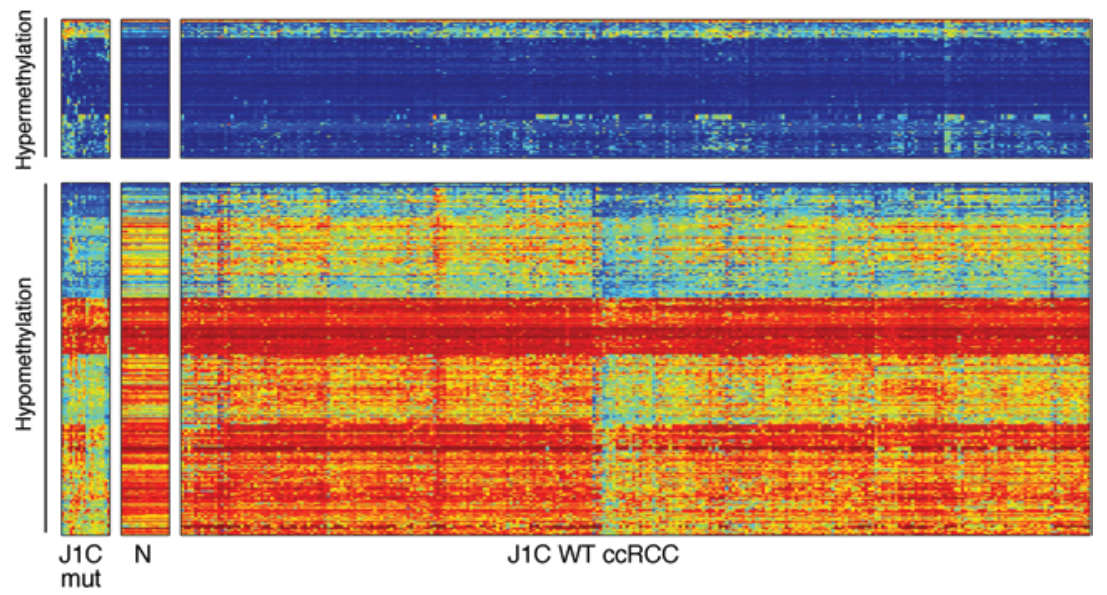

D $\square$ J1C $\square$ Others $\square$ SETD2

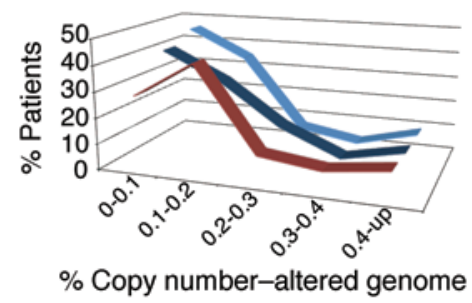

Figure 6. JARID1C-inactivating mutations cause derepression through aberrant heterochromatic transcription, enhanced genomic rearrangements, and poor prognosis in ccRCC patients. (A) Volcano plot shows a comparison of DNA methylation for JARID1C-mutated versus unmutated tumors $(n=292)$ (HumanMethylation450 platform; Illumina). Above the dashed line are indicated CpG loci with a Benjamini-Hochberg (BH) FDR of less than 0.05. Points are colored according to the FDR value. (B) Heatmap showing CpG loci with JARID1C mutation-associated (J1C mut) DNA methylation (CpG loci with an FDR $<0.05$, above the dashed line in the volcano plot); blue to red indicates low to high DNA methylation. The loci are split into those hypermethylated (top split, $n=105$ ) or hypomethylated (bottom split, $n=267$ ) in JARID1C mutants (left split) versus ccRCC WT for JARID1C (right split). The middle split shows the methylation status of 16 normal kidney samples (N). (C) Expression of human SAT2 assayed by qPCR on 9 /1C-mutated primary ccRCCs (see Supplemental Table 1 for sample information). Expression of tumor and matched normal tissue. Results are expressed relative to $R P L P O$ and are presented as the mean \pm SEM. $P<0.05$, Wilcoxon matched-pairs signed-rank test. (D) Tumors from patients with JARID1C mutations showed an increased number of CNAs ( $x$ axis corresponds to the fraction of the copy number-altered genome; $y$ axis corresponds to the frequency in patients). Data shown are from patients with JARID1C mutations, SETD2 mutations, and from CCRCC samples without SETD2 or JARID1C mutations. $P<0.05, \chi^{2}$ test over the distribution. (E) Kaplan-Meier survival curves comparing patients harboring WT with those harboring mutated JARID1C. Data were derived from TCCA (analyzed through http://www.cbioportal.org). $P=0.05$, log-rank significance test. The results shown in this figure are partly based on data generated by TCCA Research Network (http://cancergenome.nih.gov/).

indicate that JARID1C inactivation drives the unregulated expression of heterochromatic ncRNAs, which causes genomic instability and is associated with increased chromosomal rearrangements in ccRCC patients, ultimately leading to a shorter overall survival.

\section{Discussion}

In this study, we have identified a novel role for the histone demethylase JARID1C in driving heterochromatin duplication and assembly. Our data suggest that, in mammalian cells, the removal of trimethylated H3K4 by JARID1C at each cell cycle is a required step for the deposition of H3K9me3 on chromocenters, mediated by SUV39H1, and for the interaction of HP1 $\alpha$ with heterochromatin. JARID1C forms a complex with both SUV39H1 and HP1a. Co-IP experiments have also demonstrated that the adaptor protein DDB1, a homolog of yeast Rik1, belongs to this complex. Importantly, JARID1C downregulation leads to the inordinate expression of heterochromatic ncRNAs that in turn trigger increased genomic instability and, in ccRCC primary samples, is associated with more rearranged genomes and worse prognosis.
H3K4 and H3K9 trimethylation constitute mutually exclusive chromatin marks $(26,56)$. H3K9me3 is enriched on heterochromatic regions in both yeast and mammalian cells (56-58), while H3K4me3 targets transcribed euchromatin (26). H3K9me3 on heterochromatic repeat sequences prevents their transcription $(12,20)$. However, for a short interval during the $S$ phase, these sequences are accessible to RNA Pol II and are transcribed (52, 71,72 ). Of note, H3K9me3 is not consistently present on heterochromatic sequences throughout the cell cycle: its levels decrease during mitosis and remain low until the $\mathrm{S}$ phase, when they gradually increase $(52,71)$. In line with data reported in yeast and Drosophila $(27,28)$, we envision a scenario in vertebrate cells in which $\mathrm{H} 3 \mathrm{~K} 4 \mathrm{me} 3$ replaces $\mathrm{H} 3 \mathrm{~K} 9 \mathrm{me} 3$ on chromocenters during a narrow period in the $\mathrm{S}$ phase. Our data suggest that, soon after this event, removal of methyl groups by JARID1C facilitates the deposition of H3K9me3 on chromocenters by SUV39H1 and the interaction of HP1 $\alpha$ with heterochromatin, contributing to the inhibition of ncRNA transcription and, simultaneously, to heterochromatin formation, as described for Lid2 and SU(VAR)3-3 

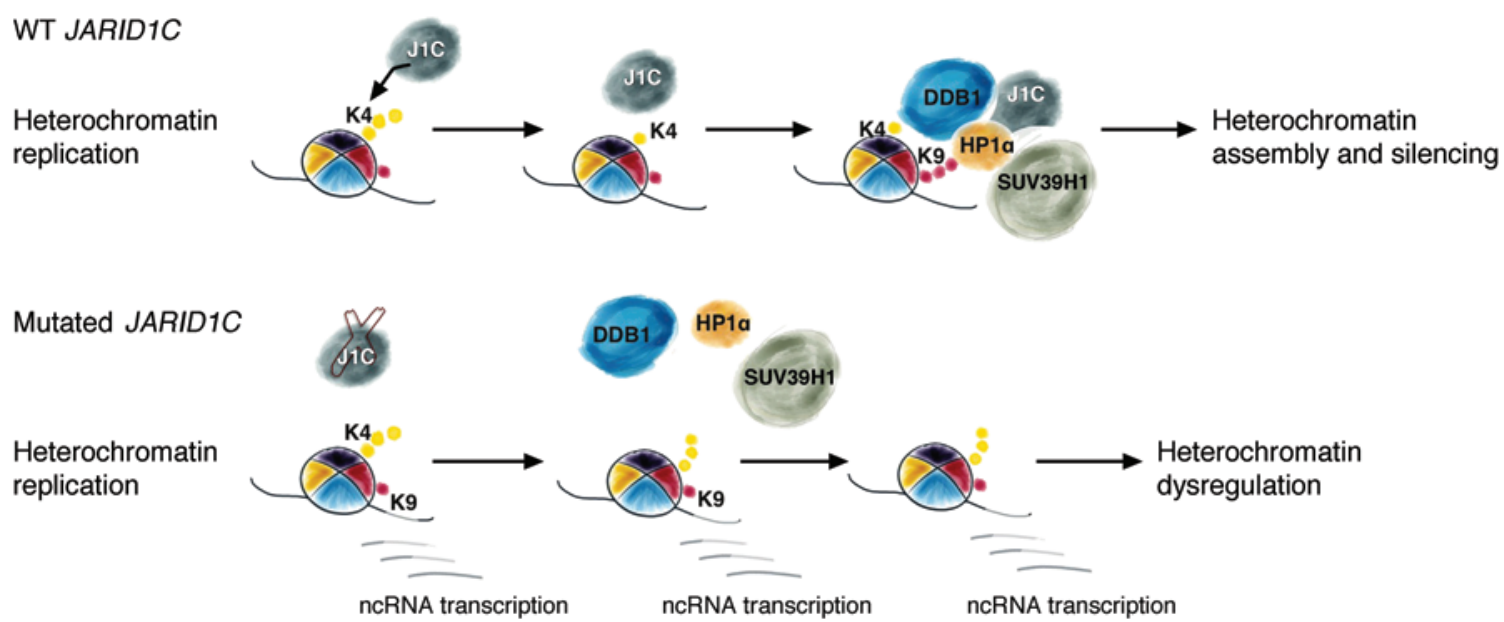

Figure 7. Proposed model for JARID1C-mediated heterochromatin assembly and silencing of ncRNAs at every cell cycle. JARID1C demethylates H3K4me3 at every cell cycle during heterochromatin duplication, thus allowing the subsequent binding of SUV39H1, trimethylation of $\mathrm{H} 3 \mathrm{~K} 9$, and $\mathrm{HP1} \alpha$ binding at satellite repeats. As a consequence, the JARID1C-SUV39H1-HP1 $\alpha$-DDB1-remodeling complex restores silencing of satellite repeats alongside heterochromatin compaction (WT JARID1C). When JARID1C is downregulated or inactivated (Mutated JARID1C), H3K4me3 levels on satellite repeats remain high, preventing SUV39H1 methyltransferase activity and HP1 $\alpha$ binding. In these conditions, persistent ncRNA transcription ensues, and heterochromatin compaction is hampered.

histone demethylases in yeast and Drosophila, respectively $(27$, 28). Intriguingly, previous in vitro studies suggested that the first of the two JARID1C PHD fingers binds trimethylated lysine 9 on histone 3 (H3K9me3) (41).

In fission yeast, a silencing complex including Dos1, Dos2, and Rik1 has been described that is crucial for engagement of the methyltransferase Crl4-SUV39H1 to nucleate heterochromatin (20, 61, 62). Human homologs for Dos1 and Dos 2 have not been described, and, indeed, we were unable to identify a plausible candidate in vertebrates on the basis of sequence and domain comparisons. On the other hand, similarities exist between the yeast Rik1, a WD- $\beta$-propeller domain-containing protein and the human adaptor protein DDB1, mostly at the structural level $(64,65)$. Rik1 is pivotal in the early phases of heterochromatin assembly $(52,66)$ and interacts with the demethylase Lid2 as well as with other proteins bound to heterochromatin including Crl4-SUV39H1 and Swi6-HP1a (27). We identified a complex in mammalian cells including DDB1, the putative Rik1 human homolog, JARID1C, SUV39H1, and HP1a, suggesting that in mammalian cells, DDB1 may participate in heterochromatin assembly in a manner similar to that of Rik1.

JARID1C has been reported in complexes including REST and co-REST (45), even if others have questioned this interaction (46). JARID1C interacts with PCNA (73) and localizes on promoters and enhancers (46). Our data support the notion that JARID1C may interact with different sets of proteins depending on the context, the cell-cycle phase, and the genomic location, as has been reported for other proteins acting on H3K4 (74).

Genomic instability is one of the core hallmarks of cancer and is an enabling feature causally associated with the acquisition of additional capabilities crucial for tumorigenesis (75). The molecular basis underlying instability is established for several hereditary cancer syndromes including, among others, $B R C A 1 / 2$, Nijmegen breakage syndrome protein 1 (NBS1), Werner syndrome helicase $(W R N)$, Bloom syndrome helicase (BLM), and Fanconi anemia (FA) genes. Germline mutations in these genes have been associated with the repair of double-strand breaks or interstrand cross links and predispose to the development of various cancers including breast and ovarian cancer, leukemias, and lymphomas. The genetic basis of instability in sporadic tumors, however, remains largely mysterious, despite clear indications of widespread, ongoing genomic instability in these cancers (76). Along these lines, it is surprising that the recent large-scale sequencing efforts, conducted in hundreds of cancer samples, have failed to identify somatic mutations targeting caretaker genes at frequencies consistent with the pervasive instability evident in tumor cells (30). On the basis of our data, we argue that, at least in some cases, genes such as JARID1C that are implicated in chromatin remodeling may be involved in crucial processes such as heterochromatin replication that, when disrupted, lead to enhanced instability. As such, these genes could be interpreted as caretakers genes, since they impinge on mechanisms that, when disrupted, jeopardize genomic integrity and trigger instability. In fact, several chromatin remodelers have been implicated in chromatin maintenance. For example, the methyltransferase SUV39H1 $(24,77)$, the ATPase complex constituents CAF-1 (18), the SWI/SNF-like protein SMARCAD (19), and the remodeler ATRX (78) restore chromatin structure after DNA replication. It should be noted, however, that with the exception of ATRX (79), genetic lesions consistently affecting these genes in cancer have not been detected thus far.

Among the potential mechanisms that could lead to instability, the disruption of heterochromatin assembly, as suggested herein as a result of JARID1C downregulation, is an intriguing avenue that cancer cells may pursue to increase their evolutionary genetic pool and to withstand the attacks mounted by the host. In fact, a recent study argued that the tumor-suppressive role of BRCA1 is rooted for the most part in an unexpected, critical activity of this gene on maintaining global heterochromatin integrity (80). As in the case of JARID1C loss, BRCA1 inactivation also leads to untimely and prolonged transcription of heterochromatic ncRNAs. These RNA species increase instability, causing multipolar spindles and unbal- 
anced sister chromatid separation $(67,80)$. This is also the case for aberrant ncRNAs in cells devoid of JARID1C. It is remarkable, then, that aberrant expression of pericentric and centromeric transcripts has been recently reported in several cancer types including pancreatic, lung, and kidney $(69,80)$.

A recent, bewildering finding in cancer genomics has been the high frequency of somatic mutations affecting genes involved in chromatin remodeling. Renal carcinoma represents a compelling case in point, since a vast array of histone modifiers have been found to be frequently inactivated, including PBRM1 (in up to $41 \%$ of patient samples analyzed) (34), UTX, SETD2, and JARID1C $(35,37)$. The prominent, causative role exerted by the inactivation of these genes on kidney tumorigenesis is reinforced by recent findings in primary human ccRCC samples (81). Both SETD2 and JARID1C presented distinct inactivating mutations in different sections of the same tumor (81). Albeit still limited in scope, as few patients were analyzed, these findings suggest that JARID1C inactivation may represent, in the subset of ccRCC where it is mutated, an obligate step toward the development and evolution of the tumor.

\section{Methods}

Cell culture. All established cell lines were purchased from American Type Culture Collection (ATCC). MEFs were prepared from C57BL/6 from E13.5 embryos. Early-passage (P2-P3) MEFs were transduced for genomic instability experiments. Cells were cultured in DMEM (NIH-3T3, HeLa, MEFs, and HEK293T) or RPMI (Caki-1, A498) supplemented with $10 \%$ FBS and antibiotics.

ChIP. Chromatin was extracted, immunoprecipitated, and used for quantitative PCR (qPCR) ChIP and ChIP-seq experiments as reported in the Supplemental Methods. ChIP-seq data have been deposited in NCBI's Gene Expression Omnibus (GEO) database (GEO GSE4452).

BrdU cytofluorimetric analysis and immunofluorescence. BrdU cytofluorimetric analysis and immunofluorescence were carried out as described in the Supplemental Methods.

Western blotting and cell fractionations. A description of the Western blotting protocol and a list of the Abs used are available in the Supplemental Methods. Isolation of the chromatin-bound cellular fraction by cytoskeleton (CSK) buffer fractionation was performed as described in the Supplemental Methods.

IP. All IP experiments were carried out in high-salt lysis buffer (20 mM Tris- $\mathrm{HCl}$ [pH 7.6], $300 \mathrm{mM} \mathrm{NaCl}, 5 \%$ glycerol, $0.25 \%$ [v/v] NP40) (ref. 82 and see Supplemental Methods). Nuclear lysates were incubated with target or isotype control Abs and protein $\mathrm{A} / \mathrm{G}$ agarose slurry beads (Calbiochem).

Generation of the JARID1C mutant. Generation of the JARID1C mutant was performed by two rounds of site-directed mutagenesis following the Stratagene protocol and using an MSCV-wtJARID1C vector as a template. Mutations introduced were H514A and A388P (41), and the mutant cDNA was defined as "mut." The primers used are listed in the Supplemental Methods.
Statistics. Averaged data were compared using 2-tailed Student's $t$ test, nonparametric Mann-Whitney $U$ test, or Fisher's exact test. To compare CTRsh and JARID1Csh distributions of cell-cycle-phase cells and the fraction of the copy number-altered genome in ccRCC patients, Pearson's $\chi^{2}$ correlation was used. Cell counts for HP1 $\alpha$, Myc-SUV39H1, bi- and trinucleated cells, and multinucleated cells were compared using Fisher's exact test. To compare the expression of human SAT2 (hSAT2) in J1C-mutated versus J1C WT ccRCCs, the Wilcoxon matched-pairs signed-rank test was used. Survival of patients with J1C WT or J1C mutations was evaluated with a log-rank significance test. Analyses were performed using GraphPad Prism 4 (GraphPad Software). A $P$ value of less than 0.05 was considered statistically significant.

Study approval. The present study of human samples was reviewed and approved by the SingHealth Centralised Institutional Review Board (Singapore). All subjects provided informed consent prior to their participation in the study.

\section{Author contributions}

BR designed the study, performed experiments, analyzed data, and wrote the manuscript. DR, EA, and DL performed experiments. MF provided experimental assistance and expertise. LM performed experiments. SS provided experimental assistance and expertise. BTT and HD provided primary ccRCC samples. KY, SBA, and RGWV performed analysis of the data set from TCGA. PAF analyzed data. LDC reviewed data and provided experimental assistance and expertise. LC analyzed data and reviewed the results. DC performed bioinformatic analysis on DNA methylation and ChIP-seq data of available TCGA KIRC data sets. GT designed the study, analyzed data, and wrote the manuscript.

\section{Acknowledgments}

We thank all the members of the Tonon laboratory for discussions and support and for critical reading of the manuscript. We also thank the Caligaris, Ghia, Muzio, Brendolan, Bernardi, and Gabellini laboratories for helpful discussions and for exchanging reagents. This work was supported by a Fondazione Italiana per la Ricerca sul Cancro (FIRC) triennial fellowship (to B. Rondinelli) and by grants from the Associazione Italiana per la Ricerca sul Cancro (AIRC) (Investigator Grants and Special Program Molecular Clinical Oncology, 5 per mille no. 9965) (to G. Tonon).

Address correspondence to: Giovanni Tonon, Functional Genomics of Cancer Unit, Division of Molecular Oncology, San Raffaele Scientific Institute, Via Olgettina 60, 20132 Milan, Italy. Phone: 39.02.2643.5624; E-mail: tonon.giovanni@hsr.it.

Beatrice Rondinelli's present address is: Department of Radiation Oncology, Dana-Farber Cancer Institute, Boston, Massachusetts, USA.
1. Jenuwein T, Allis CD. Translating the histone code. Science. 2001;293(5532):1074-1080.

2. Mills AA. Throwing the cancer switch: reciprocal roles of polycomb and trithorax proteins. Cancer. 2010;10(10):669-682.

3. Calo E, Wysocka J. Modification of enhancer chromatin: what, how, and why? Mol Cell. 2013;49(5):825-837.

4. Brown SJ, Stoilov P, Xing Y. Chromatin and epigenetic regulation of pre-mRNA processing. Hum Mol Genet. 2012;21(R1):R90-R96.

5. Ryan RJ, Bernstein BE. Molecular biology.
Science. 2012;336(6088):1513-1514.

6. Groth A, Rocha W, Verreault A, Almouzni G. Chromatin challenges during DNA replication and repair. Cell. 2007;128(4):721-733.

7. Cam HP, Chen ES, Grewal SI. Transcriptional scaffolds for heterochromatin assembly. Cell. 
2009;136(4):610-614.

8. Trojer P, Reinberg D. Facultative heterochromatin: is there a distinctive molecular signature? Mol Cell. 2007;28(1):1-13.

9. Comfort NC. From controlling elements to transposons: Barbara McClintock and the Nobel Prize. Trends Genet. 2001;17(8):475-478.

10. Durand-Dubief M, Ekwall K. Heterochromatin tells CENP-A where to go. BioEssays. 2008;30(6):526-529.

11. Naumann K, et al. Pivotal role of AtSUVH2 in heterochromatic histone methylation and gene silencing in Arabidopsis. EMBOJ. 2005;24(7):1418-1429.

12. Schotta G, et al. Central role of Drosophila SU(VAR)3-9 in histone H3-K9 methylation and heterochromatic gene silencing. $E M B O J$. 2002;21(5):1121-1131.

13. Lehnertz B, et al. Suv39h-mediated histone $\mathrm{H} 3$ lysine 9 methylation directs DNA methylation to major satellite repeats at pericentric heterochromatin. Curr Biol. 2003;13(14):1192-1200.

14. Schotta G, et al. A silencing pathway to induce $\mathrm{H} 3-\mathrm{K} 9$ and $\mathrm{H} 4-\mathrm{K} 20$ trimethylation at constitutive heterochromatin. Genes Dev. 2004;18(11):1251-1262.

15. Lindroth AM, et al. Dual histone H3 methylation marks at lysines 9 and 27 required for interaction with CHROMOMETHYLASE3. EMBO J. 2004;23(21):4286-4296.

16. Mathieu O, Probst AV, Paszkowski J. Distinct regulation of histone $\mathrm{H} 3$ methylation at lysines 27 and 9 by $\mathrm{CpG}$ methylation in Arabidopsis. ЕMBO J. 2005;24(15):2783-2791.

17. Poot RA, et al. The Williams syndrome transcription factor interacts with PCNA to target chromatin remodelling by ISWI to replication foci. Nat Cell Biol. 2004;6(12):1236-1244.

18. Quivy JP, Gerard A, Cook AJ, Roche D, Almouzni G. The HP1-p150/CAF-1 interaction is required for pericentric heterochromatin replication and S-phase progression in mouse cells. Nat Struct Mol Biol. 2008;15(9):972-979.

19. Rowbotham SP, et al. Maintenance of silent chromatin through replication requires SWI/SNF-like chromatin remodeler SMARCAD1. Mol Cell. 2011;42(3):285-296.

20. Nakayama J, Rice JC, Strahl BD, Allis CD, Grewal SI. Role of histone $\mathrm{H} 3$ lysine 9 methylation in epigenetic control of heterochromatin assembly. Science. 2001;292(5514):110-113.

21. Canzio D, et al. Chromodomain-mediated oligomerization of HP1 suggests a nucleosome-bridging mechanism for heterochromatin assembly. Mol Cell. 2011;41(1):67-81.

22. Aagaard L, et al. Functional mammalian homologues of the Drosophila PEV-modifier Su(var)3-9 encode centromere-associated proteins which complex with the heterochromatin component M31. EMBO J. 1999;18(7):1923-1938.

23. Peters AH, et al. Histone $\mathrm{H} 3$ lysine 9 methylation is an epigenetic imprint of facultative heterochromatin. Nat Genet. 2002;30(1):77-80.

24. Peters AH, et al. Loss of the Suv39h histone methyltransferases impairs mammalian heterochromatin and genome stability. Cell. 2001;107(3):323-337.

25. Lachner M, O'Carroll D, Rea S, Mechtler K,
Jenuwein T. Methylation of histone H3 lysine 9 creates a binding site for HP1 proteins. Nature. 2001;410(6824):116-120.

26. Bernstein BE, et al. Genomic maps and comparative analysis of histone modifications in human and mouse. Cell. 2005;120(2):169-181.

27. Li F, et al. Lid 2 is required for coordinating H3K4 and H3K9 methylation of heterochromatin and euchromatin. Cell. 2008;135(2):272-283.

28. Rudolph T, et al. Heterochromatin formation in Drosophila is initiated through active removal of H3K 4 methylation by the LSD1 homolog SU(VAR)3-3. Mol Cell. 2007;26(1):103-115.

29. Gopalakrishnan S, Sullivan BA, Trazzi S, Della Valle $\mathrm{G}$, Robertson KD. DNMT3B interacts with constitutive centromere protein CENP-C to modulate DNA methylation and the histone code at centromeric regions. Hum Mol Genet. 2009;18(17):3178-3193.

30. Stratton MR. Exploring the genomes of cancer cells: progress and promise. Science. 2011;331(6024):1553-1558.

31. Biegel JA, Zhou JY, Rorke LB, Stenstrom C, Wainwright LM, Fogelgren B. Germ-line and acquired mutations of INI1 in atypical teratoid and rhabdoid tumors. Cancer Res. 1999;59(1):74-79.

32. Le Gallo M, et al. Exome sequencing of serous endometrial tumors identifies recurrent somatic mutations in chromatin-remodeling and ubiquitin ligase complex genes. Nat Genet. 2012;44(12):1310-1315.

33. Dulak AM, et al. Exome and whole-genome sequencing of esophageal adenocarcinoma identifies recurrent driver events and mutational complexity. Nat Genet. 2013;45(5):478-486.

34. Varela I, et al. Exome sequencing identifies frequent mutation of the SWI/SNF complex gene PBRM1 in renal carcinoma. Nature. 2011;469(7331):539-542.

35. Dalgliesh GL, et al. Systematic sequencing of renal carcinoma reveals inactivation of histone modifying genes. Nature. 2010;463(7279):360-363.

36. Guo G, et al. Frequent mutations of genes encoding ubiquitin-mediated proteolysis pathway components in clear cell renal cell carcinoma. Nat Genet. 2012;44(1):17-19.

37. Cancer Genome Atlas Research Network. Comprehensive molecular characterization of clear cell renal cell carcinoma. Nature. 2013;499(7456):43-49.

38. Wu J, Ellison J, Salido E, Yen P, Mohandas T, Shapiro LJ. Isolation and characterization of XE169, a novel human gene that escapes X-inactivation. Hum Mol Genet. 1994;3(1):153-160.

39. Jensen LR, et al. Mutations in the JARID1C gene, which is involved in transcriptional regulation and chromatin remodeling, cause $\mathrm{X}$-linked mental retardation. Am JHum Genet. 2005;76(2):227-236

40. Rujirabanjerd S, et al. Identification and characterization of two novel JARID1C mutations: suggestion of an emerging genotype-phenotype correlation. Eur J Hum Genet. 2010;18(3):330-335.

41. Iwase $\mathrm{S}$, et al. The $\mathrm{X}$-linked mental retardation gene SMCX/JARID1C defines a family of histone $\mathrm{H} 3$ lysine 4 demethylases. Cell. 2007;128(6):1077-1088.

42. Kooistra SM, Helin K. Molecular mechanisms and potential functions of histone demethylases.
Mol Cell Biol. 2012;13(5):297-311.

43. Chen Z, et al. Structural insights into histone demethylation by JMJD2 family members. Cell. 2006;125(4):691-702.

44. Wilsker D, Patsialou A, Dallas PB, Moran E. ARID proteins: a diverse family of DNA binding proteins implicated in the control of cell growth, differentiation, and development. Cell Growth Differ. 2002;13(3):95-106.

45. Tahiliani M, et al. The histone H3K4 demethylase SMCX links REST target genes to X-linked mental retardation. Nature. 2007;447(7144):601-605.

46. Outchkourov NS, et al. Balancing of histone H3K4 methylation states by the $\mathrm{Kdm} 5 \mathrm{c} / \mathrm{SMCX}$ histone demethylase modulates promoter and enhancer function. Cell Rep. 2013;3(4):1071-1079.

47. Niu X, et al. The von Hippel-Lindau tumor suppressor protein regulates gene expression and tumor growth through histone demethylase JARID1C. Oncogene. 2012;31(6):776-786.

48. Rondinelli B, et al. H3K4me3 demethylation by the histone demethylase KDM5C/JARID1C promotes DNA replication origin firing. Nucleic Acids Res. 2015;43(5):2560-2574.

49. Ram O, et al. Combinatorial patterning of chromatin regulators uncovered by genomewide location analysis in human cells. Cell. 2011;147(7):1628-1639.

50. Maison C, Almouzni G. HP1 and the dynamics of heterochromatin maintenance. Nat Rev Mol Cell Biol. 2004;5(4):296-304.

51. Volpe T, Martienssen RA. RNA interference and heterochromatin assembly. Cold Spring Harb Perspect Biol. 2011;3(9):a003731.

52. Chen ES, Zhang K, Nicolas E, Cam HP, Zofall M, Grewal SI. Cell cycle control of centromeric repeat transcription and heterochromatin assembly. Nature. 2008;451(7179):734-737.

53. Dimitrova DS, Berezney R. The spatio-temporal organization of DNA replication sites is identical in primary, immortalized and transformed mammalian cells. JCell Sci. 2002;115(pt 21):4037-4051.

54. Papait R, Pistore C, Negri D, Pecoraro D, Cantarini L, Bonapace IM. Np95 is implicated in pericentromeric heterochromatin replication and in major satellite silencing. Mol Biol Cell. 2007;18(3):1098-1106.

55. Miao F, Natarajan R. Mapping global histone methylation patterns in the coding regions of human genes. Mol Cell Biol. 2005;25(11):4650-4661.

56. Noma K, Allis CD, Grewal SI. Transitions in distinct histone $\mathrm{H} 3$ methylation patterns at the heterochromatin domain boundaries. Science. 2001;293(5532):1150-1155.

57. Martens JH, et al. The profile of repeat-associated histone lysine methylation states in the mouse epigenome. ЕMBO J. 2005;24(4):800-812.

58. Peters AH, et al. Partitioning and plasticity of repressive histone methylation states in mammalian chromatin. Mol Cell. 2003;12(6):1577-1589.

59. Wang $\mathrm{H}$, et al. Purification and functional characterization of a histone $\mathrm{H} 3$-lysine 4-specific methyltransferase. Mol Cell. 2001;8(6):1207-1217.

60. Grewal SI, Jia S. Heterochromatin revisited. Nat Rev Genet. 2007;8(1):35-46.

61. Li F, Goto DB, Zaratiegui M, Tang X, Martienssen $\mathrm{R}$, Cande WZ. Two novel proteins, dos1 and dos2, interact with rik1 to regulate heterochro- 
matic RNA interference and histone modification. Curr Biol. 2005;15(16):1448-1457.

62. Li F, Martienssen R, Cande WZ. Coordination of DNA replication and histone modification by the Rik1-Dos2 complex. Nature. 2011;475(7355):244-248.

63. Hu J, McCall CM, Ohta T, Xiong Y. Targeted ubiquitination of CDT1 by the DDB1-CUL4A-ROC1 ligase in response to DNA damage. Nat Cell Biol. 2004;6(10):1003-1009.

64. Buscaino A, et al. Raf1 Is a DCAF for the Rik1 DDB1-like protein and has separable roles in siRNA generation and chromatin modification. PLoS Genet. 2012;8(2):e1002499.

65. Neuwald AF, Poleksic A. PSI-BLAST searches using hidden markov models of structural repeats: prediction of an unusual sliding DNA clamp and of beta-propellers in UV-damaged DNA-binding protein. Nucleic Acids Res. 2000;28(18):3570-3580.

66. Allshire RC, Nimmo ER, Ekwall K, Javerzat JP, Cranston G. Mutations derepressing silent centromeric domains in fission yeast disrupt chromosome segregation. Genes Dev. 1995;9(2):218-233.

67. Bouzinba-Segard H, Guais A, Francastel C. Accumulation of small murine minor satellite transcripts leads to impaired centromeric architecture and function. Proc Natl Acad Sci U S A.
2006;103(23):8709-8714.

68. Probst AV, Okamoto I, Casanova M, El Marjou F, Le Baccon P, Almouzni G. A strand-specific burst in transcription of pericentric satellites is required for chromocenter formation and early mouse development. Dev Cell. 2010;19(4):625-638.

69. Ting DT, et al. Aberrant overexpression of satellite repeats in pancreatic and other epithelial cancers. Science. 2011;331(6017):593-596.

70. Cerami E, et al. The cBio cancer genomics portal: an open platform for exploring multidimensional cancer genomics data. Cancer Discov. 2012;2(5):401-404.

71. Kloc A, Zaratiegui M, Nora E, Martienssen R. RNA interference guides histone modification during the $S$ phase of chromosomal replication. Curr Biol. 2008;18(7):490-495.

72. Zaratiegui M, et al. RNAi promotes heterochromatic silencing through replication-coupled release of RNA Pol II. Nature. 2011;479(7371):135-138.

73. Liang Z, Diamond M, Smith JA, Schnell M, Daniel R. Proliferating cell nuclear antigen is required for loading of the SMCX/KMD5C histone demethylase onto chromatin. Epigenetics Chromatin. 2011;4(1):18.

74. van Nuland R, Smits AH, Pallaki P, Jansen PW, Vermeulen M, Timmers HT. Quantitative dissection and stoichiometry determination of the human SET1/MLL histone methyltransferase complexes. Mol Cell Biol. 2013;33(10):2067-2077.

75. Hanahan D, Weinberg RA. Hallmarks of cancer: the next generation. Cell. 2011;144(5):646-674.

76. Negrini S, Gorgoulis VG, Halazonetis TD. Genomic instability - an evolving hallmark of cancer. Mol Cell Biol. 2010;11(3):220-228.

77. Loyola A, et al. The HP1 $\alpha$-CAF1-SetDB1-containing complex provides H3K9me1 for Suv39mediated K9me3 in pericentric heterochromatin. EMBO Rep. 2009;10(7):769-775.

78. Ritchie K, Seah C, Moulin J, Isaac C, Dick F, Berube NG. Loss of ATRX leads to chromosome cohesion and congression defects. JCell Biol. 2008;180(2):315-324.

79. Zhang J, et al. Whole-genome sequencing identifies genetic alterations in pediatric low-grade gliomas. Nat Genet. 2013;45(6):602-612.

80. Zhu Q, et al. BRCA1 tumour suppression occurs via heterochromatin-mediated silencing. Nature. 2011;477(7363):179-184.

81. Gerlinger $\mathrm{M}$, et al. Intratumor heterogeneity and branched evolution revealed by multiregion sequencing. N EnglJ Med. 2012;366(10):883-892.

82. Vella P, et al. Tet proteins connect the O-linked $\mathrm{N}$-acetylglucosamine transferase Ogt to chromatin in embryonic stem cells. Mol Cell. 2013;49(4):645-656. 